\title{
APT Tip Shape Modifications During Analysis, Its Implications, and the Potential to Measure Tip Shapes in Real Time via Soft-X-Ray Ptychography
}

\author{
Paul van der Heide ${ }^{1 *}$, Igor Mathotkin ${ }^{1,2}$, Wilfried Vandervorst ${ }^{1,2}$ and Claudia Fleischmann ${ }^{1}$ \\ 1. IMEC, Kapeldreef 75, Leuven, Belgium \\ 2. Instituut voor Kern- en Stralingsfysica (IKS), KU Leuven, Celestijnenlaan 200D, Leuven, Belgium. \\ * Corresponding author: Paul.vanderheide@imec.be
}

Since the turn of the $21^{\text {st }}$ century, Atom Probe Tomography (APT) has opened materials characterization possibilities beyond that thought possible [1]. The semiconductor industry is another field experiencing progress beyond that previously imagined [2]. With this, the latest R\&D efforts are now pushing for the ability to characterize next generation heterogeneous nano-structures in $3 \mathrm{D}$ to atomic resolution and with all atoms identifiable, a capability that, in principle, APT can provide.

APT can be thought of as a bridge between Transmission Electron Microscopy (TEM) and Secondary Ion Mass Spectrometry (SIMS). The maturatization of TEM and SIMS has resulted in these being accepted as part of the analytical tool box applied in the semiconductor industry. The same cannot be said for APT. This stems primarily from issues with quantification and image distortion. This report concerns the topic of image distortion, and how these could be measured in real time.

Image distortions can be related back to the APT tip shape, or more precisely geometric deviations from the idealized spherical tip shape that is not accounted for in image reconstruction algorithms. Tip shape deviations can arise from a) preferential evaporation from the side of the tip facing the laser when lower wavelength (UV) lasers are used (this is noted on homogeneous materials [3]), and/or b) preferential evaporation of atoms from regions within heterogeneous structures displaying differential evaporation probabilities [4]. Spherical tip shape deviations modify the extraction field above the tip, thereby altering the trajectories of evaporated atoms, which are ionized as they depart the tip. This in turn alters the recorded distribution relative to that from a spherical tip. If not accounted for, this will translate into a distorted final reconstructed image. One means to account for this is to determine the APT tip shape, and ultimately in real time.

Although recent advances have allowed for observations of the APT tip shape modification via electron microscopies [5] or Scanning Probe Microscopies (SPM) [6], there is at the time of preparing this text, difficultly in measuring APT tip shape modification occurring during APT analysis. A major obstacle to this lies in the high fields used in APT, as this will, to some extent, distort the trajectories in charged particle imaging capabilities. Specifically engineered APT-systems with in-situ TEM or SPM capabilities may provide solutions in this respect.

An alternative solution could lie in the use of soft x-ray/extreme UV photons from a tabletop High Harmonic Generator (HHG). Indeed, the use of a coherent light source would allow for 2D lens-less imaging using coherent diffraction imaging (CDI) or it's modification referred to as ptychography [7]. If ported into an APT instrument combined with the needed 2D detectors and the computational capability required for phase retrieval, one can then envisage a set up illustrated in figure 1. The next question then raised is whether the HHG source, which is pulsed, could also act to induce evaporation under the high field conditions used in APT, i.e. to act as the source for both APT and APT tip imaging.

To test the feasibility of APT tip imaging via ptychography, a series of studies on dummy scaled-up APT 
tips were collected under zero field conditions at the I13-1 DIAMOND [8] beam line using $12.4 \mathrm{keV} \mathrm{x-}$ rays along with the software based on the sPIE algorithm for ptychography [9].

The first results shown in figure 2, where SEM and ptychography images are compared, reveals that the APT tips do survive over the needed acquisition times and that the acquisition times were also acceptable, i.e. of the order of minutes. Note: For practical reasons, the beamline configuration was not optimized for ptychography, therefore ultimate resolution was not attained. However, even in the non-optimized configuration used, the phase image of the tip shown in figure 2 does show an overall shape and distinguishable material contrast consistent with the actual structure. Next steps will be to explore advantages and complications of soft X-ray CDI/ptychography in order to understand the true in-lab reachable spatial resolution limits on APT tips.

References:

[1] MK Miller and RG Forbes in "Atom-Probe Tomography: The Local Electrode Atom Probe", (Springer, New York).

[2] http://www.economist.com/technology-quarterly/2016-03-12/after-moores-law

[3] S Koelling et al., Journal of Applied Physics 109 (2011), p. 104909.

[4] D Melkonyan et al., Ultramicroscopy 197 (2017), p. 100.

[5] M Herbig, Scripta Materialia 148 (2018), p. 98.

[7] C Fleischmann et al., Ultramicroscopy 194 (2018), p. 221.

[7] A Hitchcock, Journal of Electron Spectroscopy and Related Phenomena 200 (2015), p. 40.

[8] D Batey et al., Microscopy and Microanalysis 24(S2) (2018), p. 40.

[9] D Batey et al., Phys. Rev. A. 89 (2014), p. 043812.

[10] I Makhotkin et al., to be published.

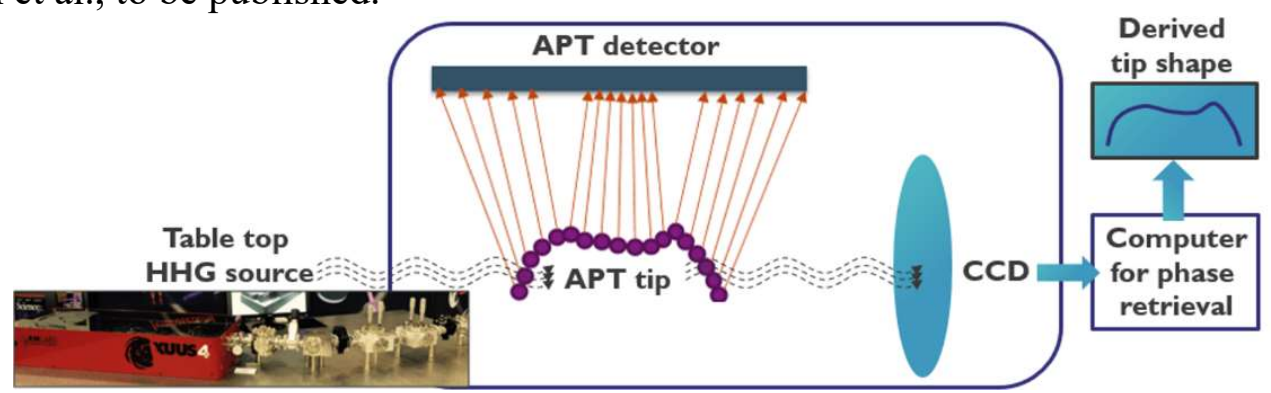

Figure 1. Concept of real time APT tip imaging using a table top HHG source for producing a coherent photon beam as required to carry out ptychography.

a)

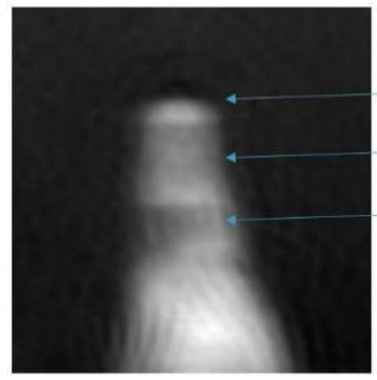

b)

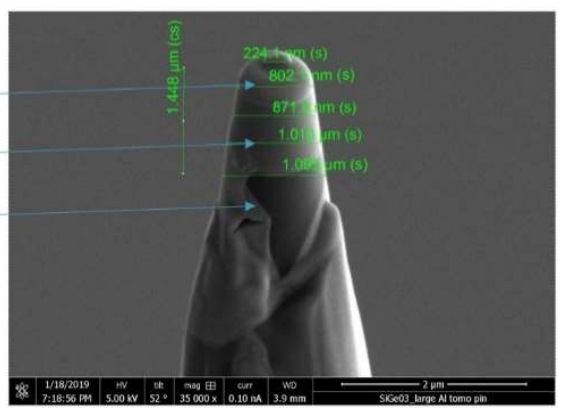

Figure 2. Images of a scaled up APT tip collected via a) ptychography and b) SEM (see text) [10]. 